\title{
Diseño e implementación de una Interfaz de Control para la Integración de una Celda de Manufactura Flexible
}

\section{Design and implementation of a Control Interface for the Integration of a Flexible Manufacturing Cell}

\author{
ROJAS-GARNICA, Juan Carlos†*, MUÑOZ-MATA, José Lorenzo, RANGEL-ROMERO, Carlos y \\ OCOTITLA-MUÑOZ, Alma Delia
}

\section{Universidad Tecnológica de Puebla}

ID $1^{\text {er }}$ Autor: Juan Carlos, Rojas-Garnica / ORC ID: 0000-0002-2261-587X, CVU CONACYT ID: 66417

ID $1^{\text {er }}$ Coautor: José Lorenzo, Muñoz-Mata / ORC ID: 0000-0001-7813-5579, CVU CONACYT ID: 177117

ID $2^{\text {do }}$ Coautor: Carlos, Rangel-Romero / ORC ID: 0000-0003-4879-4228, CVU CONACYT ID: 894477

ID $3^{\text {er }}$ Coautor: Alma Delia, Ocotitla-Muñoz / ORC ID: 0000-0002-3266-3226

\section{Resumen}

En la industria moderna los sistemas de manufactura flexible (SMF) son indispensables para el incremento en la productividad y en la calidad de los productos. Las celdas que los constituyen cuentan con varias estaciones de trabajo conectadas entre sí a través de un protocolo de comunicación, por lo tanto, con lo cual son capaces de identificar y distinguir entre las diferentes partes o productos procesados en el sistema. Además, tienen la capacidad de ajustarse a la demanda de procesar diferentes productos y cantidades. En los SMF se utilizan protocolos de comunicación tales como Device Net, Ethernet, entre otros. Sin embargo, existen casos en los que no se cuenta con módulos de comunicación, por lo que es necesario desarrollar dispositivos alternos para comunicar entre sí las estaciones de trabajo para integrar una celda de manufactura flexible que cuente con las características necesarias para ser considerada como tal. En este trabajo se presentan los resultados obtenidos del estudio de infraestructura para el desarrollo de una interfaz de comunicación de una celda de manufactura flexible utilizando un microcontrolador, con la capacidad de comunicar dos estaciones de trabajo. En este caso particular, un robot FANUC M6iB con un centro de maquinado HAAS VF2.

Sistemas de Manufactura Flexible, Robot Industrial, CNC

\begin{abstract}
In modern industry, flexible manufacturing systems (FMS) are indispensable to increase productivity and product quality. The cells that constitute them have several work stations connected to each other through a communication protocol, therefore, with which they are able to identify and distinguish between the different parts or products processed in the system. Furthermore, they have the ability of adaptation to the demand of processing different products and quantities. In the FMS communication protocols are used such as Device Net, Ethernet, among others. However, there are cases in which there are no communication modules; however, it is necessary to develop alternative devices to communicate the work stations with each other to integrate a flexible manufacturing cell with the necessary characteristics. This paper presents the results obtained from the study of infrastructure for the development of a communication interface to integrate a flexible manufacturing cell using a microcontroller, with the capability of communicating two work stations. In this particular case, a FANUC M6iB robot with a HAAS VF2 machining center.
\end{abstract}

Flexible Manufacturing System, Industrial Robot, CNC

Citación: ROJAS-GARNICA, Juan Carlos, MUÑOZ-MATA, José Lorenzo, RANGEL-ROMERO, Carlos y OCOTITLAMUÑOZ, Alma Delia. Diseño e implementación de una Interfaz de Control para la Integración de una Celda de Manufactura Flexible. Revista del Diseño Innovativo. 2019 3-9: 23-28

\footnotetext{
* Correspondencia del Autor (Correo electrónico: carlos.rojas@ utpuebla.edu.mx)

$\dagger$ Investigador contribuyendo como primer autor.
} 


\section{Introducción}

Los sistemas de manufactura flexible (SMF) son los más seleccionados en la industria debido a las numerosas ventajas, tales como: baja cantidad de material en almacenamiento, alta competitividad, alta calidad de producción, bajo tiempo de fabricación y bajo costo, los cuales han sido conceptualizados desde los años 60's (Emin 2015). Los SMF se pueden definir como estaciones de trabajo controladas por computadora y manejo de materiales. Son un tipo de celda con equipos automatizados que aplica la tecnología de grupos, el cual contiene conglomerados de estaciones de trabajo con diversos procesos, interconectados por un sistema automático de carga, almacenamiento y descarga de materiales (Siwamogsatham 2007). Es flexible, porque es capaz de procesar varios tipos de enseres a diferentes cantidades, que pueden ser ajustadas en respuesta a los comportamientos de una determinada demanda (Groover 2001).

El SMF debe tener la habilidad para identificar y distinguir entre las diferentes partes o productos procesados por el sistema. Contar con un rápido cambio de las instrucciones de operación y un rápido cambio de la configuración física. La flexibilidad es un atributo que aplica a los sistemas manuales y automatizados (Kang 2016).

Para que un sistema sea considerado flexible debe satisfacer las siguientes pruebas: variedad de partes, cambio de programación, recuperación de errores y de nuevas partes.

Los SMF se emplean protocolos de comunicación industrial, tales como: DeviceNet, Ethernet, Profibus, Fieldbus, entre otros (Guerrero et al. 2009).

Pero existen situaciones, en los que no se cuenta con dicha infraestructura éstos llegan a ser demasiado costosos para su implementación, por lo que es necesario implementar medios alternativos para la comunicación entre estaciones de trabajo para lograr una celda de manufactura flexible que cuente con las características necesarias de tal manera que pueda ser considerada como tal. Los SMF se clasifican por el nivel de flexibilidad y el número de máquinas (Singh 2018). Si el sistema tiene una estación de trabajo se le denomina celda de una sola máquina (CSM).
Si contiene de dos a tres estaciones de trabajo tales como una máquina de control numérico, un robot $y$ un sistema de almacenamiento, se le denomina celda de manufactura flexible (CMF) (Hernández 2012).

En este trabajo se presenta el desarrollo de una interfaz de comunicación para implementar una CMF como desarrollo de infraestructura para propósitos académicos y docencia dentro de la Universidad Tecnológica de Puebla. El sistema se implementó con el uso de un microcontrolador configurado como Controlador Lógico Programable (PLC).

La interfaz, tiene la capacidad de comunicar entre sí un robot FANUC M6iB y un centro de maquinado HAAS VF2, utilizando una comunicación de señales simples, con la ventaja inherente de ser un dispositivo mucho más barato de implementar que un PLC.

\section{Desarrollo Experimental}

El diagrama a bloques del sistema a implementar se muestra en la Figura 1, para lograr una CMF integrando un robot y un centro de maquinado, para lo cual debemos tener en consideración los siguientes aspectos.

Se utilizó un microcontrolador (PIC16F877A Microchip) (Microchip 2006) por sus prestaciones para el desarrollo de la interfaz de comunicación. Además, se diseñaron circuitos aislantes para manejar niveles de voltaje industriales, con el propósito de que dicha interfaz funcione como un PLC.

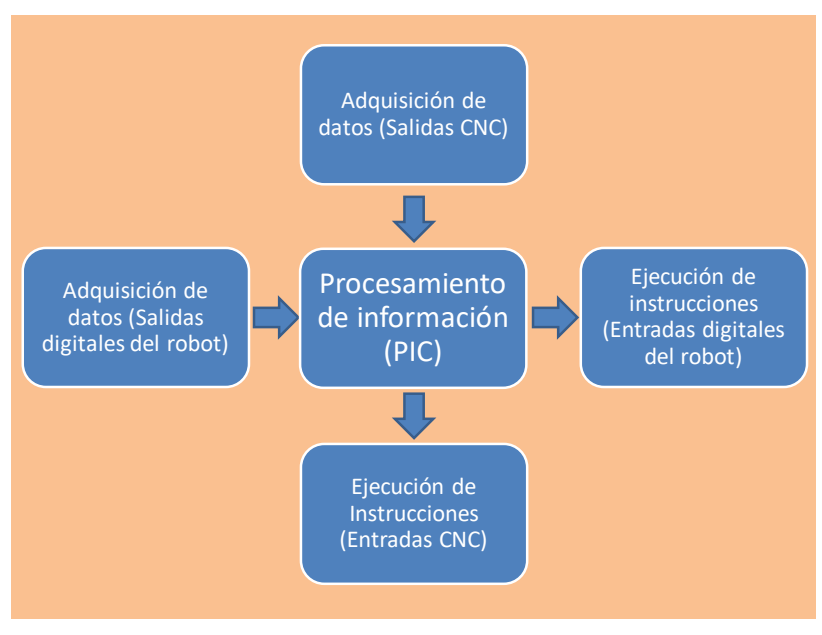

Figura 1 Diagrama a bloques del sistema implementado Fuente: Elaboración Propia 


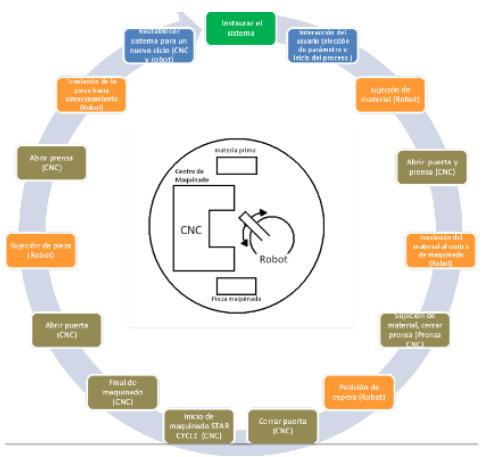

Figura 2 Diagrama del ciclo de la celda de manufactura flexible

Fuente: Elaboración Propia

Para la implementación de la CMF, es necesario el uso de al menos dos estaciones de trabajo, las cuales consisten de un robot FANUC Mi6B (FANUC Robotics 2006), un centro de maquinado HAAS (Haas Automation Inc. 2016) y un sistema de manejo de materiales. La secuencia de funcionamiento para la CMF debe realizar una serie de etapas de acuerdo con el diagrama a bloques propuesto de la Figura 2. Se crea una comunicación bidireccional entre el robot y el centro de maquinado, a través de la interfaz. El dispositivo está conectado directamente a los módulos de entradas y salidas del robot. La interfaz envía señales a los módulos y el robot ejecute las tareas indicadas de acuerdo con el algoritmo del proceso de manufactura. $\mathrm{Al}$ mismo tiempo, la interfaz se comunica con el $\mathrm{CNC}$ a través de señales obtenidas desde el panel de control del mismo. Además, se colocaron sensores en las puertas del centro de maquinado para monitorear su estado y por ende poder manipular la apertura o cierre de las mismas. Se utilizó una prensa neumática para la sujeción del material dentro del centro de maquinado con la particularidad de que puede ser controlada manualmente o a través de la tarjeta de interfaz.

Para lograr lo anterior es necesario realizar un estudio del funcionamiento de los módulos de entrada/salida (E/S) del robot, así como también del centro de maquinado para establecer comunicación y control con el mismo.

\section{Adquisición y envío de señales de la tarjeta hacia el robot}

El desarrollo de la comunicación entre el robot y la interfaz se implementó utilizado los módulos de E/S del robot FANUC Mi6B, con su controlador R-Ji3B, que cuenta con 5 ranuras para base horizontales modelo ABU05A.
Las cuales están dedicadas para un tipo de salidas y entradas digitales determinadas para un propósito específico. En el caso particular de este proyecto se utilizó el módulo de entradas digitales AID32E1 y el módulo de salidas digitales AOD32D1 (GE FANUC Automation 2000).

La propuesta del circuito implementado consistió en aislar la electrónica con los módulos de E/S del robot utilizando optoacopladores. Para comunicarse con el CNC se utilizaron relevadores para asegurar un total aislamiento con dicho dispositivo.

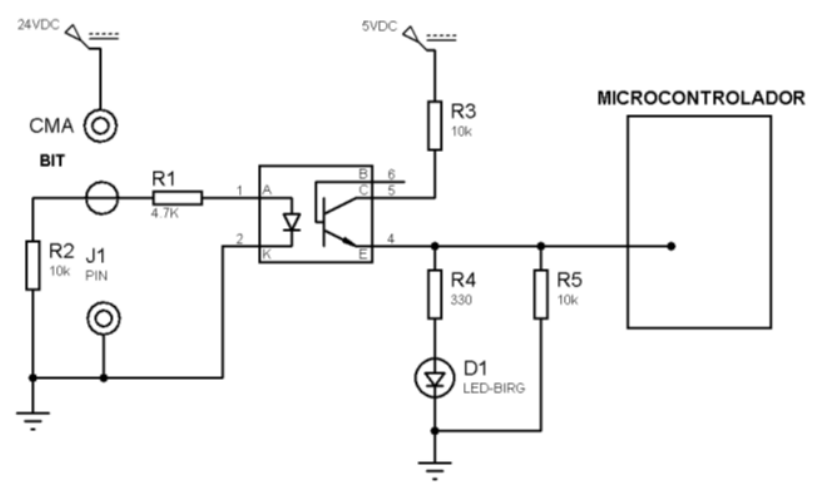

Figura 3 Circuito de aislamiento para adquisición de datos Fuente: Elaboración Propia

En la Figura 3 se muestra el diagrama del circuito diseñado para la recepción de datos. Como se puede observar es un circuito simple, con el cual se realiza la obtención de la señal proveniente del robot de $24 \mathrm{~V}$ y es convertida a $5 \mathrm{~V}$ a través de los optoacopladores.

En la Figura 4, se observa el circuito diseñado correspondiente al envío de datos. Dicho circuito genera las señales desde la interfaz convirtiendo en un nivel de voltaje de 5 $\mathrm{V}$ hacia el robot con un voltaje de $24 \mathrm{~V}$.

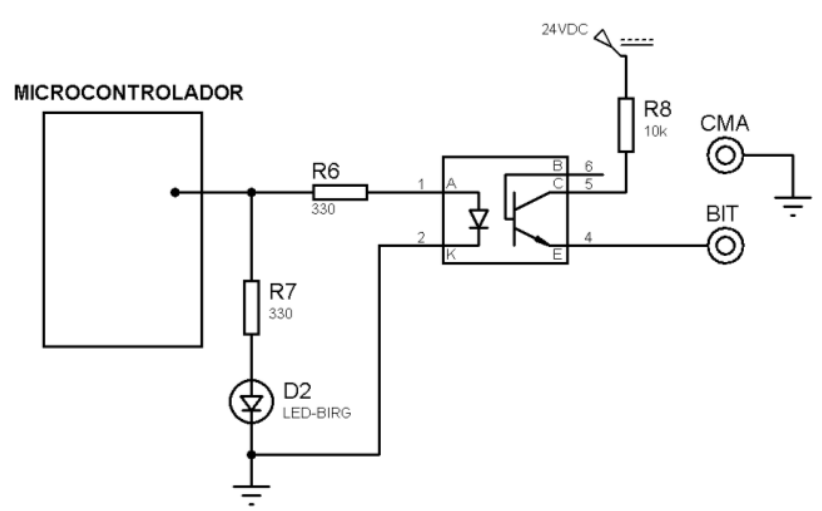

Figura 4 Circuito de aislamiento para envío de datos Fuente: Elaboración Propia 
Estas propuestas fueron implementadas y simuladas utilizando software de simulación de diseño asistido por computadora. Es importante señalar que el sistema debe ser inteligente, es decir, que se debe conocer el estado del sistema a través de sensores o de señales para saber si el sistema tiene algún problema de funcionamiento. En el caso del robot se toman como señales de monitoreo las señales provenientes del sensor, es decir, si el robot no responde cada vez que realice una tarea, el algoritmo del microcontrolador será capaz de detener el ciclo del proceso para realizar el proceso manual de revisión al sistema.

Para realizar la comunicación con el centro de maquinado se intervino el tablero de control del dispositivo, dado que solo cuenta con entradas y salidas de propósito general a través del protocolo de comunicaciones DeviceNet. Por lo anterior, se implementaron circuitos utilizando relevadores para lograr aislar completamente el tablero de control. En la Figura 5 se muestra la distribución de los sensores de apertura y cierre de la puerta, así como el circuito de acoplamiento conectado a la interfaz. Se colocaron sensores en las puertas del centro de maquinado para conocer su estado lógico, pues es necesario conocer el estado de estas según el algoritmo del ciclo de la celda de manufactura, manipularlas y de ser necesario detener el proceso.

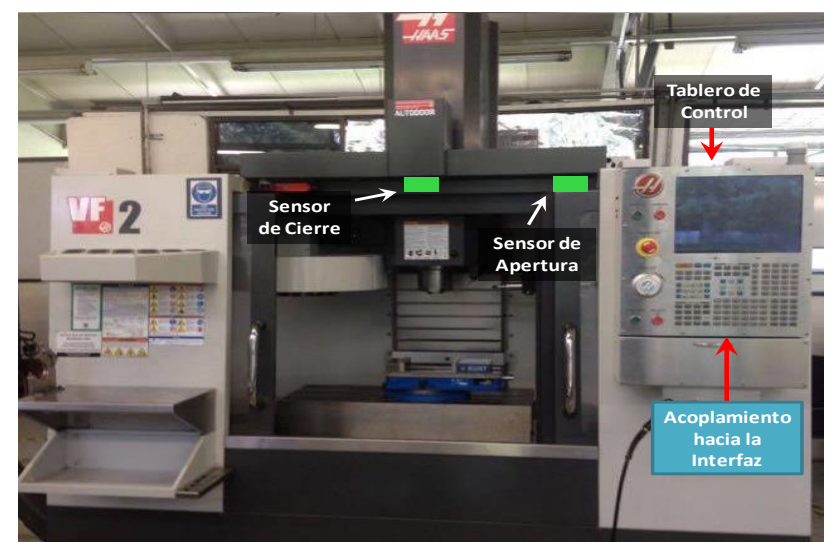

Figura 5 Distribución de sensores y acoplamento en el CNC

El software para el desarrollo de la interfaz fue el compilador CCS (Trejo 2009), el cual contiene el algoritmo para la comunicación del ciclo de trabajo de la celda de manufactura e integrar el robot y el centro de maquinado, los cuales son la infraestructura disponible para la realización de dicho proyecto. El diagrama de flujo del firmware desarrollado se muestra en la Figura 6.

\section{Resultados obtenidos}

Una vez realizadas las simulaciones y pruebas correspondientes se procedió a desarrollar el circuito impreso de la tarjeta de interfaz. La tarjeta terminada se muestra en las Figura 7.

La tarjeta cuenta con diez entradas digitales de las cuales seis están dedicadas a las señales provenientes del robot, dos señales dedicadas a las puertas del CNC y dos para conocer el estatus de la prensa. Además, cuenta con diez señales de salida dentro de las cuales seis están dedicadas a enviar señales al robot, dos señales dedicadas al CNC (para abrir puertas e iniciar ciclo de maquinado) y dos para abrir y cerrar la prensa neumática.
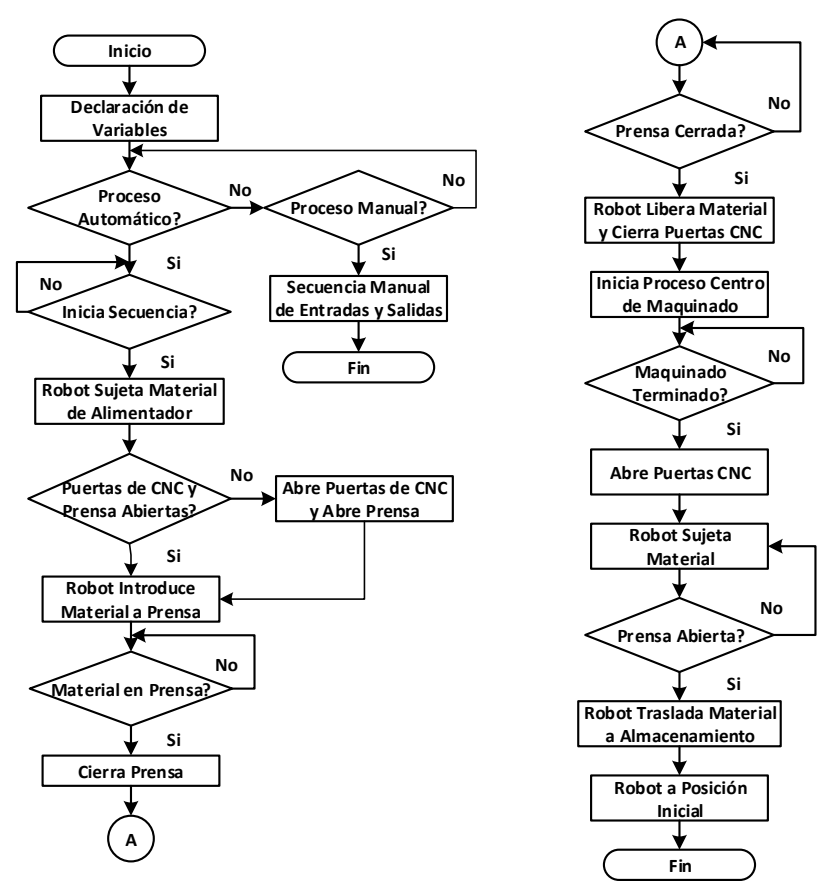

Figura 6 Diagrama de flujo del firmware para la interfaz implementada

Fuente: Elaboración Propia

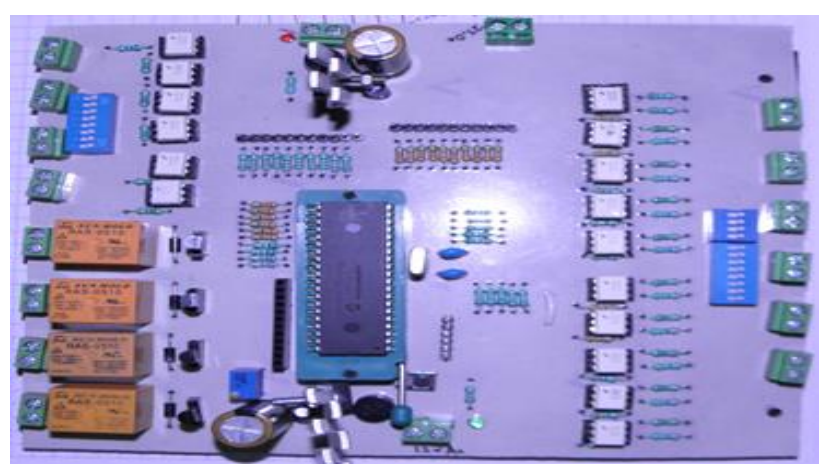

Figura 7 Tarjeta de la interfaz de comunicaciones

Se realizaron las pruebas correspondientes a la tarjeta ya terminada y se instaló dentro del gabinete del controlador del robot como se muestra en las Figura 8.

ROJAS-GARNICA, Juan Carlos, MUÑOZ-MATA, José Lorenzo, RANGEL-ROMERO, Carlos y OCOTITLA-MUÑOZ, Alma Delia Diseño e implementación de una Interfaz de Control para la Integración de una Celda de Manufactura Flexible. Revista del Diseño Innovativo. 2019 
Después de un conjunto de pruebas con el robot se aseguró el correcto funcionamiento de la tarjeta. Finalmente, se realizó la integración de la tarjeta con el robot y el CNC logrando implementar un proceso de manufactura flexible completo, donde los resultados fueron satisfactorios.
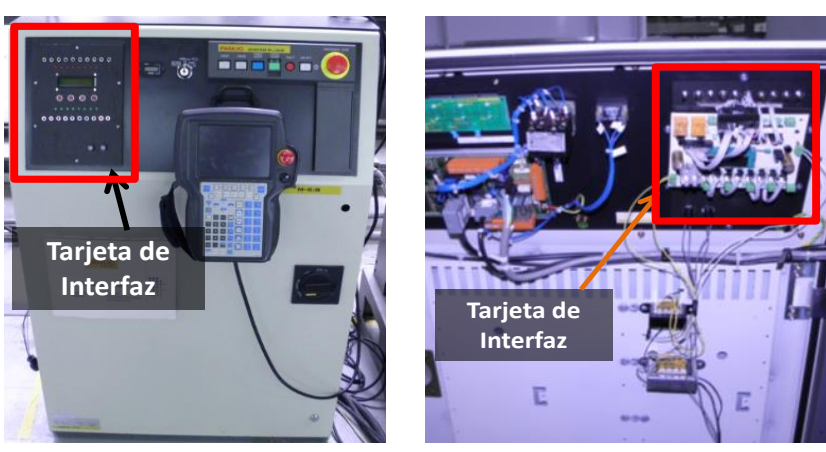

Figura 8 Tarjeta instalada dentro del gabinete del robot Fanuc

El maquinado realizado, para corroborar el correcto funcionamiento de la CMF consistió en un proceso simple de careo de una pieza de aluminio, tomando en cuenta que el proceso puede ser modificado de manera sencilla ya que la infraestructura es flexible para el cambio de proceso de manufactura. En la Figura 9 se muestra la integración de la CMF implementada.

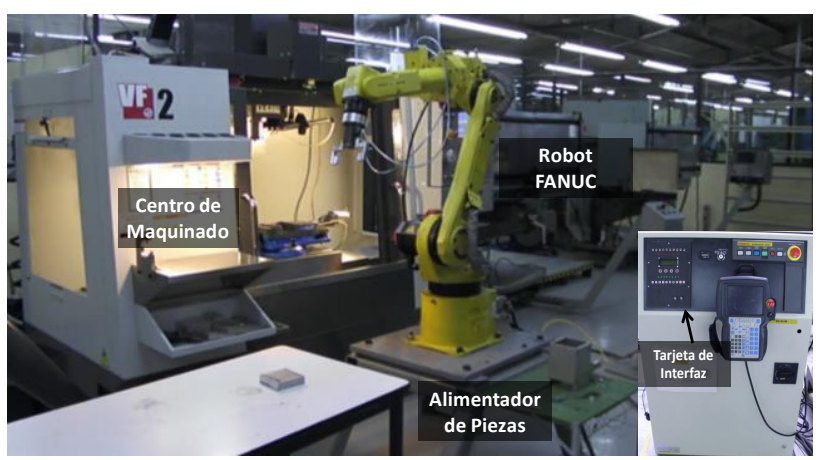

Figura Integración de la Celda de Manufactura Flexible Fuente: Elaboración Propia

A continuación, se muestran las etapas del proceso de manufactura de la CMF implementada en el laboratorio de manufactura de la Universidad Tecnológica de Puebla de acuerdo con el algoritmo desarrollado en la Figura 6.

Al comenzar el proceso, la interfaz solicita el inicio del mismo y el robot se coloca en la posición inicial. Posteriormente, solicita si el proceso es automático o manual. Si el proceso es manual, se ejecuta la secuencia de prueba para verificar si el sistema funciona correctamente en cada una de ellas.
Si se selecciona el proceso automático, el algoritmo pregunta por el inicio del mismo. Enseguida, el robot toma el material del alimentador. Se verifica si las puertas del CNC y la prensa están abiertas, de lo contrario se ejecuta la apertura de las mismas. El robot coloca la pieza en la prensa y detecta si la pieza está sobre la prensa. Se ejecuta el cierre de prensa y se detecta si la acción es realizada. El robot libera la pieza y queda en espera mientras se realiza el proceso de maquinado. En la Figura 10 se muestra la secuencia de la primera parte del proceso de manufactura descrito.

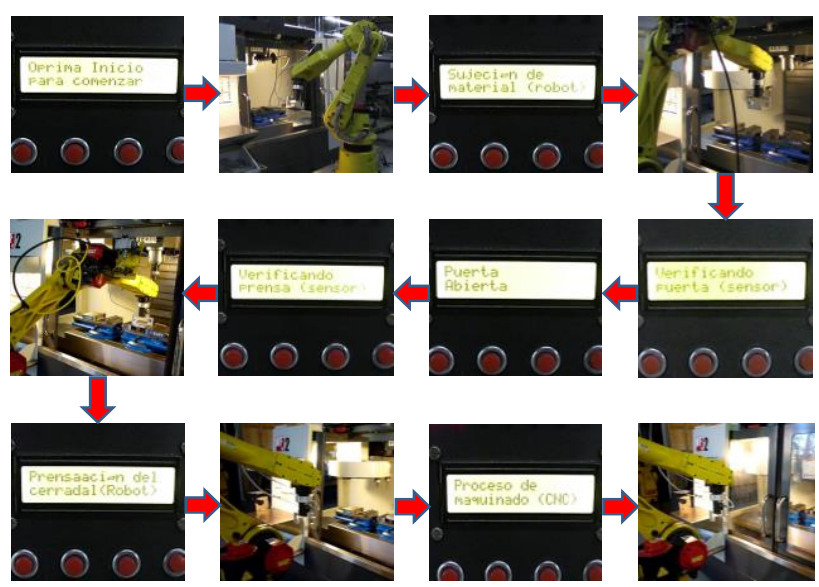

Figura 10 Diagrama de la primera parte de la secuencia del proceso de la CMF

Fuente: Elaboración Propia

Una vez terminado el proceso de maquinado el CNC abre las puertas para que el robot realice la sujeción del material. Se realiza la apertura de la prensa y se verifica si la misma ha sido abierta. Finalmente, el robot traslada el material a su almacenamiento, libera el material y posteriormente el robot ejecuta la rutina para colocarse en su posición inicial para un nuevo proceso. En la Figura 11 se muestra el diagrama de la secuencia de la segunda parte del proceso de manufactura.

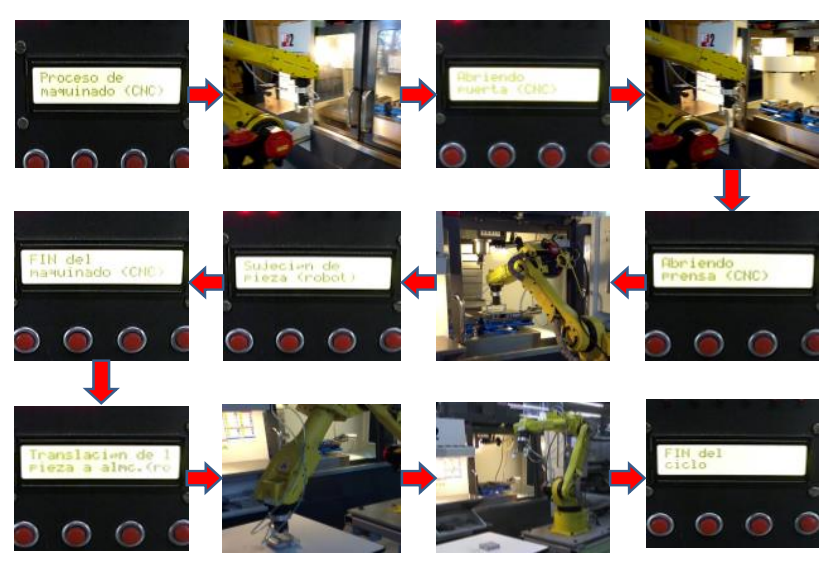

Figura 11 Diagrama de la segunda parte de la secuencia del proceso de la CMF

Fuente: Elaboración Propia

ROJAS-GARNICA, Juan Carlos, MUÑOZ-MATA, José Lorenzo, RANGEL-ROMERO, Carlos y OCOTITLA-MUÑOZ, Alma Delia Diseño e implementación de una Interfaz de Control para la Integración de una Celda de Manufactura Flexible. Revista del Diseño Innovativo. 2019 
Al realizar las pruebas correspondientes del proceso de manufactura, se ha podido comprobar el correcto funcionamiento en la implementación y el desarrollo de la CMF. La interfaz desarrollada funciona de manera satisfactoria. Además, la comunicación con las estaciones de trabajo funciona adecuadamente, por lo que se puede decir que el sistema desarrollado tiene un buen desempeño. Con el desarrollo de la CMF se ha podido mejorar la infraestructura con la que se cuenta en el laboratorio de la Universidad Tecnológica de Puebla, con la cual se podrá consolidar los conocimientos en temas relacionados con los SMF.

\section{Agradecimientos}

Se agradece las facilidades prestadas para la integración de la celda a la Universidad Tecnológica de Puebla.

\section{Conclusiones}

Se ha realizado el estudio del funcionamiento de los módulos de Entrada/Salida del robot FANUC M6iB, así como el desarrollo de circuitos de prueba para comprobar el correcto funcionamiento de estos.

Se han propuesto circuitos para el aislamiento de los módulos del robot y el centro de maquinado para alcanzar la integración de una CMF a través de la tarjeta de interfaz desarrollada.

Con el desarrollo de este proyecto se ha logrado desarrollar infraestructura para el fortalecimiento de conocimientos y experiencia, orientados a los sistemas mecatrónicos en el área de manufactura flexible y sus aplicaciones, tanto para el estudiante como para el profesorado.

\section{Trabajo Futuro}

Como trabajo futuro se está trabajando en una nueva versión de la tarjeta de interfaz, así como en la actualización del firmware del microcontrolador para hacer más robusto el funcionamiento de la celda de manufactura flexible.

\section{Referencias}

A. Singh, J. Singh, M. Ali, (2018). Some Control Strategies in a Flexible Manufacturing SystemA Simulation Perspective. International Journal of Applied Engineering Research, 7, 5296-5303.
FANUC Robotics America, Inc. (2006). FANUC Robotics R-J3iB and R-J3iC Controller, M-6iB, M-6iB/6S, Mechanical Unit and Maintenance Manual 3900 W. Hamlin Road Rochester Hills, Michigan.

G. Hernández (2012). Fundamentos de Control Inteligente de La Manufactura Flexible ( $1^{\mathrm{a}}$ ed.). España: Eae Editorial Academia Española.

García Trejo, E. (2009). Compilador C CCS y Simulador Proteus para Microcontroladores PIC ( $2^{\mathrm{a}}$ ed.). Ciudad de México, México: Alfaomega.

GE Fanuc Automation Europe (2000) I/O Unit model a connection maintenance manual [Manual], Featherstone Road http://ucc.colorado.edu/fanuc/61813e.pdf

H. S. Kang, J. Y. Lee, S. S. Choi, H. Kim. J. H. Park, J. Y. Son, B. H. Kim, S. D. Noh, (2016). Smart Manufacturing: Past Research, Present Findings, and Future Directions. International Journal of Precision Engineering and Manufacturing-Green Technology, 3(1), 111128.

Haas Automation Inc. (2016 1 febrero). Mill Operator's Manual [Manual], https://aemqas.haascnc.com/content/dam/haasc nc/en/service/manual/operator/english---milloperator\%27s-manual---2016.pdf.

M. Emin, A. Atmaca, (2014). Implementation of an Overall Design of a Flexible Manufacturing System. Procedia Technology, 19, 185-192.

M. P. Groover, (2001). Automation, Production Systems and Computer-Integrated Manufacturing (4ta ed.). New York, USA: Pearson.

Microchip. (2006, 1 enero). PIC18F2455/2550/ 4455/4550 Data Sheet [Datasheet]. Recuperado 10 octubre, 2017, de https://ww1.microchip.com /downloads/en/devicedoc/39632c.pdf

T. Siwamogsatham, C. Saygin (2007). Auctionbased distributed scheduling and control scheme for flexible manufacturing systems. International Journal of Production Research, $42,547-572$.

V. Guerrero, L. Martínez, R. Yuste (2009). Comunicaciones Industriales (1a ed.). Ciudad de México, México: Alfaomega-Marcombo.

ROJAS-GARNICA, Juan Carlos, MUÑOZ-MATA, José Lorenzo, RANGEL-ROMERO, Carlos y OCOTITLA-MUÑOZ, Alma Delia. Diseño e implementación de una Interfaz de Control para la Integración de una Celda de Manufactura Flexible. Revista del Diseño Innovativo. 2019 